\title{
Analysis of Green Environmental Protection Concept in Packaging Design
}

\author{
Bing Liu ${ }^{1,}$, Ruiqiu Pang 2, b \\ ${ }^{1}$ Academy of Fine Arts, Northeast Normal University, Changchun130024, China \\ ${ }^{2}$ School of Geographical Sciences, Northeast Normal University, Changchun130024, China \\ a ice8515@163.com, br rq56789@yeah.net
}

\begin{abstract}
Based on the environmental sustainable development, combining the concept of green environmental protection and the development of social civilization, the design principle and material application of environmental protection packaging are analyzed. The research shows: the use of environmentally friendly materials, moderate and appropriate packaging, and the flexible use of environmental protection green concept, can effectively solve waste of resources and environmental pollution, designers should start from their own responsibilities and obligations, integrated a variety of factors, the implementation and promotion of environmentally friendly packaging design and development.
\end{abstract}

Keywords: Packaging Design; Green Environmental Protection Concept; Sustainable development

\section{Introduction}

With the rapid development of social productive forces, human beings have created enormous material wealth, but also brought a series of environmental crisis, the depletion of resources and the destruction of ecology have restricted the sustainable development of economy, people's consumption concept and living habits also received different challenges. The emergence of environmental crisis, people's awareness of environmental protection gradually enhanced, this kind of green environmental protection consciousness has a direct impact on people's consumption concept, the concept of green environmental protection arises at the historic moment [1].

People tend to choose the material environmental protection, recyclable and reusable packaging design, and the exclusion of visual pollution and easy to produce environmental pollution packaging. In order to cater to the concept of consumers, businesses from the material selection, design concepts have also begun to consider the green environmental factors, began the development of green packaging. The prevalence of green packaging culture promoted the rise of green packaging design

\section{Analyses of Packaging Design and Green Environmental Protection Concept}

\subsection{Analysis of Packing Design}

Packaging refers to the general name of containers, materials and auxiliary materials used for the protection of products, convenient storage and transportation, and promotion of sales. Also refers to combination of art and technology, the use of appropriate materials, shapes, text, graphics, and protection technology, such as the creation of organic packaging entities, to shape the image of goods. Packaging design refers to the use of appropriate packaging materials, the use of clever means of technology, for the goods of the container structure design and packaging of the decorative design, it includes three elements: appearance, composition and material, its function in: one to protect the physical function of products, such as the protection of products, easy to store in transportation and other functions; two, to convey the brand information business functions, that is, through the packaging design, arouse the desire of consumers to buy, so that consumers have a motive to buy [2-4].

\subsection{Analysis on the Green Environmental Protection Packaging Design}

Green packaging design is to follow the international "3R+1D" principle of packaging: "3R" means Reduce, Resuse and Recycle, "1D" means Degradable. Green packaging design focuses on the ecological balance between man and nature, in the design of each link are fully considered, minimize 
the damage to the environment, in the selection of materials as far as possible the use of green materials [5].

Environmentally friendly commodity packaging also refers to the use of renewable, recyclable, non-polluting materials on the packaging of goods; with the following functions: portable function, protection, the function of transmitting commodity information, preservation and storage function, easy to use, action and management functions.

\subsection{The Importance and Necessity of Environmental Protection Packaging Design}

With the prosperity and development of economy and commodity market, the phenomenon of luxury packaging and disposable waste habits, resulting in a large number of packaging wastes; these wastes not only pollute the environment, but also consume a large amount of energy. To solve this problem, we need to reform the packaging materials, priority to the use of environmentally friendly materials, and now the packaging design and the materials used in the realization of the function of the product to meet the needs of users, must be in the product and consumption of the two major aspects to assume the important responsibility of saving resources and protecting the environment [6].

Green packaging has been paid more and more attention, its scope is gradually expanding, people began to pay attention to the packaging of the humanities value and its long-term business and social value. In packaging design, we must establish the moral concept of green environmental protection design, choose non-toxic harmless, easy to decompose material, the whole process of design is green, ecological and humanized, and the combination of material and spirit, to contribute to the natural environment and sustainable development.

In today's modernization, environmentally friendly packaging is a matter of everyone's daily life; environmental protection packaging for human beings, future generations and the contribution of the entire human society is immeasurable. Environmentally friendly packaging design will become the future direction of packaging design.

\section{Analyses of Principles of Green Design for Packaging Design}

\subsection{Application of Packaging Materials}

Green packaging design should start with the packaging materials, green environmental protection material is a kind of material with good environmental compatibility on the premise of satisfying the basic function of packaging, and select the material should pay attention to the following points.

Preferred energy consumption, low pollution materials. For example, paper materials, it is a kind of material that is widely used in packaging industry, the raw material of paper is mainly natural plant fiber, which can be decomposed quickly in nature, caused by a low degree of environmental pollution, and can be recycled.

Preferred renewable materials, such as recycled paper, recycled plastics, recycled glass, etc., as far as possible the use of recyclable materials; improve resource utilization, sustainable development.

Preferred material with good environmental compatibility, to avoid the use of toxic, harmful, radioactive materials, the materials used should be easy to recycle, reuse, remanufacturing or easy degradation.

Materials used in packaging design, we should vigorously promote the use of environmentally friendly ecological packaging materials, for example, the use of glass as a packaging material, the glass can be recycled and reused, it is recyclable packaging materials, the method of glass recycling refers to the waste glass material after the acquisition of waste, it is regenerated by physical and chemical methods so that it can be reused again. Paper packaging, is now the most common use of a packaging material, the paper has its environmental characteristics, its own composition is the plant fiber, discarded into the natural environment can be quickly decomposed.

Materials used in packaging design, adhere to the principle of "easy recovery and recycling", the purpose of recycling is to make the old packaging waste after a certain technical treatment, as raw materials, into the new products, not only can save resources and energy, but also can reduce the pollution of the environment. For example, recycled aluminum beverage bottle packaging, re melting aluminum, beer bottles, milk bottles etc., can be also recycled, this kind of packaging has a long life 
cycle and little negative impact on the environment, in the packaging design of the environment, it is worth promoting.

Packaging design materials, adhere to the "material can be degraded in nature, do not pollute the environment" principle, for example "White pollution", plastic packing box, disposable tableware, foaming material, plastic beverage bottle, plastic outer package of various food and various plastic outer packing bag, a large number of plastic packaging materials which are difficult to degrade in nature, the environmental pollution caused by plastic waste is difficult to eliminate, to solve this problem, it is necessary to reduce the use of such plastic PVC products, develop new packaging materials to replace the material difficult to degrade [7].

\subsection{Application of Moderate and Appropriate Packaging}

The environmental protection design of commodity packaging should give full consideration to the structural design of packaging, that is, the simplification of packaging structure, excessive packaging of goods will consume a large amount of materials, resulting in waste of resources, so that the increase in the number of urban garbage, too gorgeous packaging waste of resources, aggravate environmental pollution. Only by changing the structure of the packaging, reducing the cost of packaging, improving the structural strength of the packaging, reducing the risk of damage in the transport of goods, reducing the repeated application of packaging materials, in order to reduce the cost of packaging [8].

Excessive packaging, resulting in environmental pollution cannot be ignored; people must pay attention to it. In developed countries, the problem of excessive packaging has been included in the national laws and regulations, to limit its harm, Germany announced the "circular economy law", Denmark implemented a "green tax" system, many national laws and regulations, the production and sale of packaging manufacturers, the responsibility of the packaging of waste materials recycling.

\subsection{Application of Environmental Protection and Green Concept}

The development of packaging design is accompanied by the development of human civilization, in the social environment of mechanical chemical industry, and constantly adapt to the development of market economy, the packaging design, its function is no longer just to meet the commodity transport and protection of the role of goods, packaging should not only have a higher aesthetic, but also have a certain cultural heritage and cultural connotations, packaging is an important way for enterprises to deliver commodity information and business philosophy. On the information transmission of packaging design, should emphasize and promote environmental protection information, in the material, pattern, color, text reveals the green environment, it can not only reflect the concept of environmental protection, but also create a sense of trust and identity.

Green design has been widely welcomed by consumers, the application of green environmental protection materials, will give consumers a wealth of visual and tactile experience, the use of natural raw materials can be recognized by consumers, we should fully understand the physical properties of natural materials, protect the environment, allow consumers to obtain material and spiritual satisfaction.

In the exploration and use of environmentally friendly materials on the road, We should make full use of its characteristics, from the concept of environmental protection, the needs of consumers, the concept of modern design, the inheritance and innovation of national culture, etc., the choice and the use, manufacture conforms to the green environmental protection request, but also has the unique characteristic packing design, to meet the needs of modern consumers, with strong market competitiveness, and to meet the requirements of modern society, it will be of great value to change the appearance of the whole packaging market.

\section{Conclusions}

Today, with the booming market economy, packaging industry plays an important role in economic life, the development of the times has promoted the continuous development of packaging technology, to promote the concept of green environmental protection, and designers have the primary task. In future commodity packaging design, packaging designers must understand the material and 
environmental sustainability, in the packaging design of the time to establish the concept of green design, choose non-toxic harmless, easy decomposition of the material, the whole process of design to achieve green, ecological, and contribute to the sustainable development of the natural environment; designers should have a strong sense of social responsibility, all design from the perspective of protecting the environment and resources, make their own design and the trend of the times has always been synchronized, at the same time, do a good job in packaging design, to protect the environment for the healthy development of mankind to make due contributions.

\section{Corresponding Author}

Ruiqiu Pang, Associate professor, Northeast Normal University, CHINA, rq56789@yeah.net.

\section{References}

[1] Maria Assunta Barchiesi, Silvia Castellan, Roberta Costa: In the Eye of the Beholder: Communicating CSR Through Color in Packaging Design, Journal of Marketing Communications, Vol. 8 (2016), p. 1-14.

[2] Li Yinxing: Sustainable Development of Green Packaging Design, Packaging Engineering, Vol. 35 (2014), p. 73-76.

[3] Zhang Lile: Green Packaging Design under the New Era of Green Psychology, Packaging Engineering, Vol. 37 (2016), p. 72-75.

[4] F. Nitsche, I. Reiche, J. Stewart \& S. Whittingham: European technical guide on package design safety reports for transport packages containing radioactive material, Packaging, Transport, Storage \& Security Of Radioactive Material, Vol. 19 (2013), p. 87-93.

[5] L Wang, M Yang: Green Packaging Design on the Principle of 3R, Packaging Engineering, Vol. 29 (2008), p.162-165.

[6] Justine A. Spack, Virginia E. Board, Lindsay M. Crighton, Phillip M. Kostka, James D. Ivory: It's Easy Being Green: The Effects of Argument and Imagery on Consumer Responses to Green Product Packaging, Environmental Communication, Vol. 6 (2012), p.441-458.

[7] Zhang Lilei, Qiao Jie: Green Design of Visual Language in Product Packaging Design, Packaging Engineering, Vol. 36 (2015), p. 26-28.

[8] Shun Rao, Jing Liu: Research on the Modern Packaging Design Paradigm under the Background of Low Carbon and Green Thinking Orientation, 3rd International Symposium on Engineering Technology, Education and Management (ISETEM), Guangzhou, PEOPLES R CHINA, NOV 12-13 (2016), p. 57-62. 